\title{
An image-guided femoroplasty system: Development and initial cadaver studies
}

\author{
Yoshito Otake $^{\mathrm{a}^{*}}$, Mehran Armand ${ }^{\mathrm{b}, \mathrm{c}}$, Ofri Sadowsky ${ }^{\mathrm{a}}$, Robert S. Armiger ${ }^{\mathrm{b}}$, Michael D. Kutzer ${ }^{\mathrm{b}}$, \\ Simon C. Mears ${ }^{\mathrm{c}}$, Peter Kazanzides ${ }^{\mathrm{a}}$, Russell H. Taylor ${ }^{\mathrm{a}}$ \\ ${ }^{a}$ Department of Computer Science, the Johns Hopkins University, \\ 3400 N. Charles St., Baltimore, MD, USA 21218 \\ ${ }^{\mathrm{b}}$ Applied Physics Laboratory, the Johns Hopkins University, \\ 11100 Johns Hopkins Rd., Laurel, MD, USA 20723 \\ ${ }^{\mathrm{c}}$ Department of Orthopaedic Surgery, Johns Hopkins Bayview Medical Center, \\ 4940 Eastern Ave., Baltimore, MD, USA 21224
}

\begin{abstract}
This paper describes the development and initial cadaver studies using a prototype image-guided surgery system for femoroplasty, which is a potential alternative treatment for reducing fracture risk in patients with severe osteoporosis. Our goal is to develop an integrated surgical guidance system that will allow surgeons to augment the femur using patient-specific biomechanical planning and intraoperative analysis tools. This paper focuses on the intraoperative module, which provides real-time navigation of an injection device and estimates the distribution of the injected material relative to the preoperative plan. Patient registration is performed using intensity-based 2D/3D registration of X-ray images and preoperative CT data. To co-register intraoperative X-ray images and optical tracker coordinates, we integrated a custom optically-tracked fluoroscope fiducial allowing real-time visualization of the injection device with respect to the patient's femur. During the procedure, X-ray images were acquired to estimate the 3D distribution of the injected augmentation material (e.g. bone cement). Based on the injection progress, the injection plan could be adjusted if needed to achieve optimal distribution. In phantom experiments, the average target registration error at the center of the femoral head was $1.4 \mathrm{~mm}$ and the rotational error was 0.8 degrees when two images were used. Three cadaveric studies demonstrated efficacy of the navigation system. Our preliminary simulation study of the 3D shape reconstruction algorithm demonstrated that the 3D distribution of the augmentation material could be estimated within $12 \%$ error from six X-ray images.
\end{abstract}

Keywords: Image-guided therapy, bone augmentation, osteoporosis, 2D/3D registration, space carving

\section{INTRODUCTION}

We are investigating femoroplasty as an alternative treatment for patients with severe osteoporosis and who have previously suffered a fracture of the contralateral hip. Although this procedure has yet to be tested clinically, experimental results [1][2] show that in reinforced cadaveric femurs, the maximum load applied prior to fracture was increased by $43 \%$ relative to the native bone, which suggests that the femoroplasty has the potential to reduce the risk of fracture in an osteoporotic hip. The objective of this treatment is to reduce the risk of osteoporotic fracture, however, without precise geometrical and mechanical planning based on analysis of the structural strength and stress distributions, adding augmentation material may have the potential downside of developing stress concentrations between augmented and native bone [3]. Hence, we believe that preoperative planning based on patient-specific biomechanics and precise intraoperative navigation is critical to the success of femoroplasty. To this end, we are developing an image-guided surgery system for enhancing accuracy and efficiency of femoroplasty. In this system, the image guidance assists the surgeon in controlling the hand-held injection device. The system consists of 4 subsystems: 1) a preoperative plan optimization system using CT based patient-specific finite element modeling [4]; 2) a hand-held robotic injection device which is used to precisely control the volume and rate of delivery of augmentation material; 3) an intraoperative navigation system for guiding the surgeon to operate the hand-held injection device precisely according to the planned

* otake@jhu.edu; phone 1-410-516-5396; fax 1-410-516-4410; JSPS Postdoctoral Fellow for Research Abroad

Medical Imaging 2010: Visualization, Image-Guided Procedures, and Modeling, edited by Kenneth H. Wong, Michael I. Miga, Proc. of SPIE Vol. 7625, 76250P - ( 2010 SPIE · CCC code: 1605-7422/10/\$18 - doi: 10.1117/12.845458 


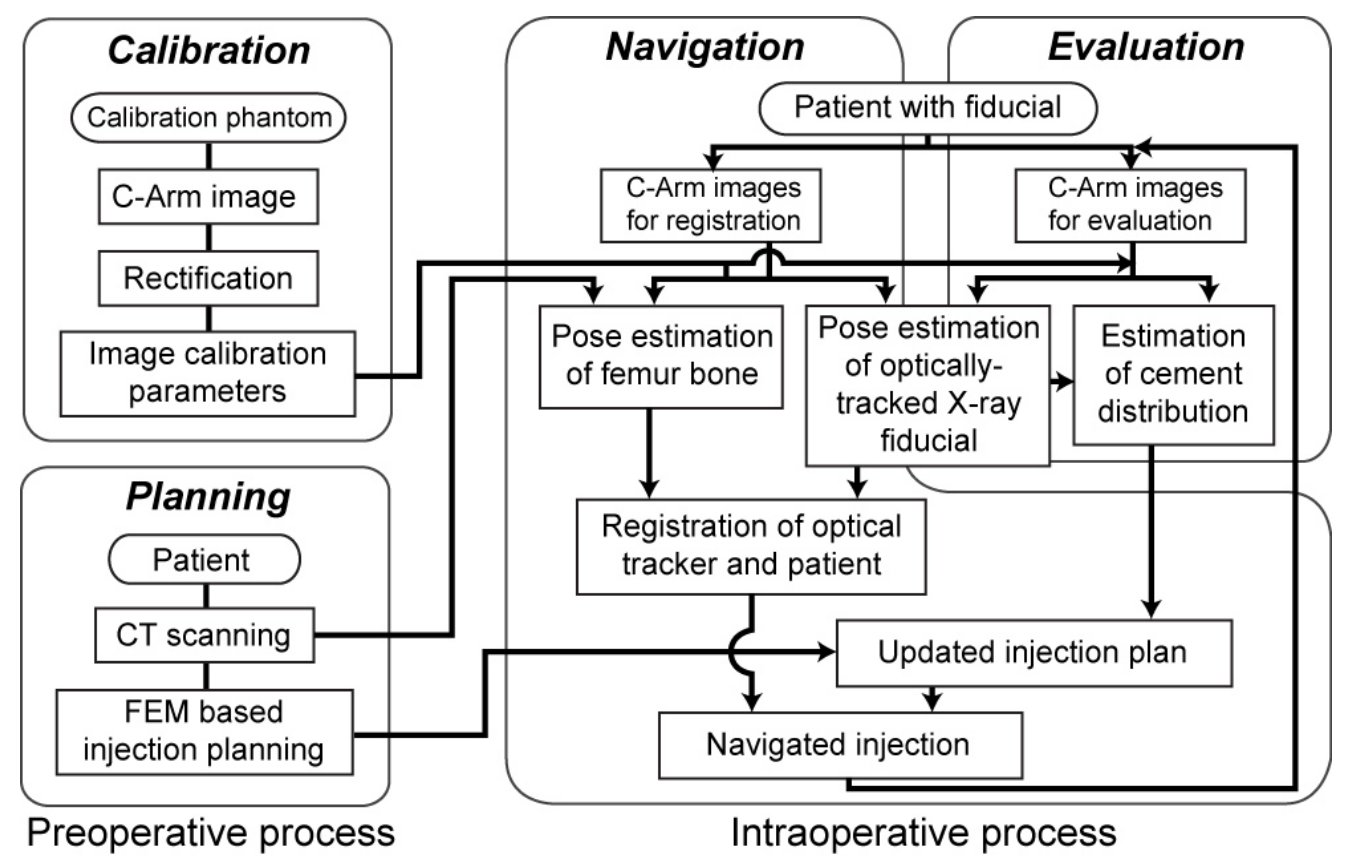

Figure 1. Block diagram for the robotic-assisted bone augmentation system

trajectory, 4) an intraoperative evaluation system for measuring the distribution of the injected augmentation material and enabling the injection plan to be updated as the surgery progresses. This paper focuses on the intraoperative navigation and evaluation aspects of the system (items 3 and 4).

First, in order to implement the intraoperative navigation system (item 3), we extended a previously reported methodology [5][6] for registration using a custom optically-tracked fluoroscope fiducial and evaluated the accuracy of the device. Second, to achieve accurate estimation of the distribution of the augmentation material while minimizing the amount of exposure of ionizing radiation to the patient and surgical staff, we developed a method to reconstruct the 3D shape of the injected augmentation material from only a few 2D X-ray projection images. A shape estimation method using silhouette-based space curving is reported. Finally, the feasibility, accuracy and potential efficacy of the system are examined in preliminary studies involving three cadaver specimens.

\section{METHODS}

The image-guided femoroplasty process is divided into four major steps, Planning, Calibration, Navigation and Evaluation (Figure 1). In the planning step, an injection plan including a path of the injection tool and the volume of injection is created based on an optimization with finite-element analysis. In the calibration step, the focus is to estimate the geometry of the X-ray source and detector of the C-Arm device as well as its distortion parameters. Overall system configuration and coordinate system relationships are shown in Figure 2. In the navigation stage, registration of the preoperative CT coordinate system and optical tracker coordinate system is performed using the fluoroscope tracking fiducial, which we call FTRAC [6]. During the injection, the hand-held injection device is guided using an optical tracker. In the evaluation phase, several additional X-ray images are acquired and the 3-dimensional distribution of the injected augmentation material is estimated from these images. The results of the evaluation phase are then used to update the preoperative plan for subsequent injections. The planning step has been described previously in [4], and the following subsections describe the remaining steps in detail.

\subsection{Preoperative calibration}

In the preoperative calibration stage, two types of calibration were conducted: 1) calibration of the C-Arm, and 2) calibration of the custom optical-tracking tools.

1) C-Arm calibration 


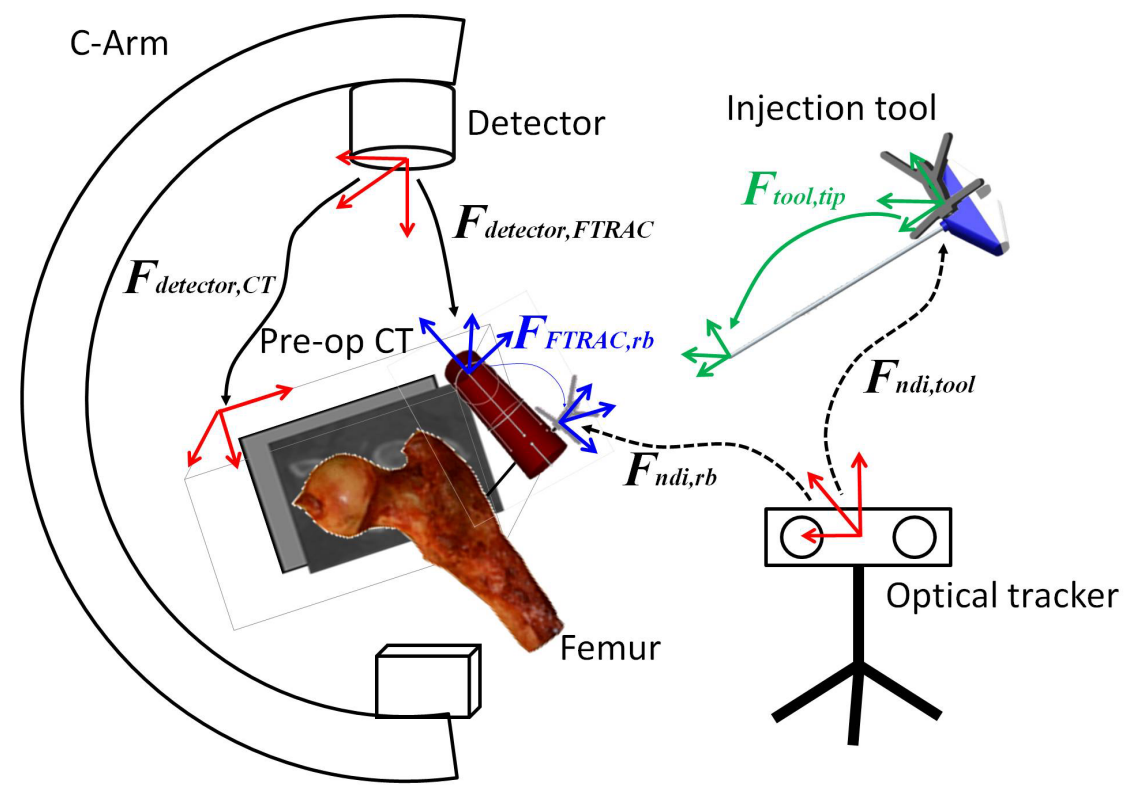

Figure 2. System configuration and registration chain. Solid arrows mark preoperatively calibrated transformations, dashed arrows mark transformations that are updated during injection. An arrow from coordinate system $a$ to coordinate system $b$ denotes the transformation $F_{a, b}$

C-Arm calibration involves image distortion correction and intrinsic projection parameter estimation. The C-Arm calibration process used an X-ray image of a custom designed calibration phantom (discussed in our previously published work [7]). The phantom included a wire grid for distortion correction, and two planes with radiopaque diamond patterns for determining the intrinsic parameters. All the images in the following steps were rectified using the distortion parameters calculated from this phantom. The estimated intrinsic parameters were used in the registration process described in 2.2 .

2) Optical-tracking tool calibration

Two custom optical-tracking tools were used in the proposed system. One was the X-ray fluoroscope tracking fiducial incorporated with the optical-tracking rigid body, which we called hybrid-fiducial (Figure 3(a)) and the other one was an injection device (Figure 3(b)). The hybrid-fiducial was designed to achieve the registration between patient CT coordinate and optically-tracked rigid body using 2D X-ray projection image. The transformation between the rigid body coordinate system $\left(F_{r b}\right)$ (see Figure 2$)$ and the FTRAC coordinate $\left(F_{F T R A C}\right)$ of the hybrid fiducial was preoperatively measured by CT. The optically-tracked injection device was calibrated in two steps: pivot calibration of the tip position and axis calibration of the injection cannula (Figure 4). Using these calibrations, the transformation between the optically-tracked rigid body and the needle tip coordinate system $\left(F_{\text {tool,tip }}\right.$ in Figure 2$)$ was computed. Figure 4 (c) and (d) shows the recorded data during calibration. From the data, $F_{\text {tool,tip }}$ was
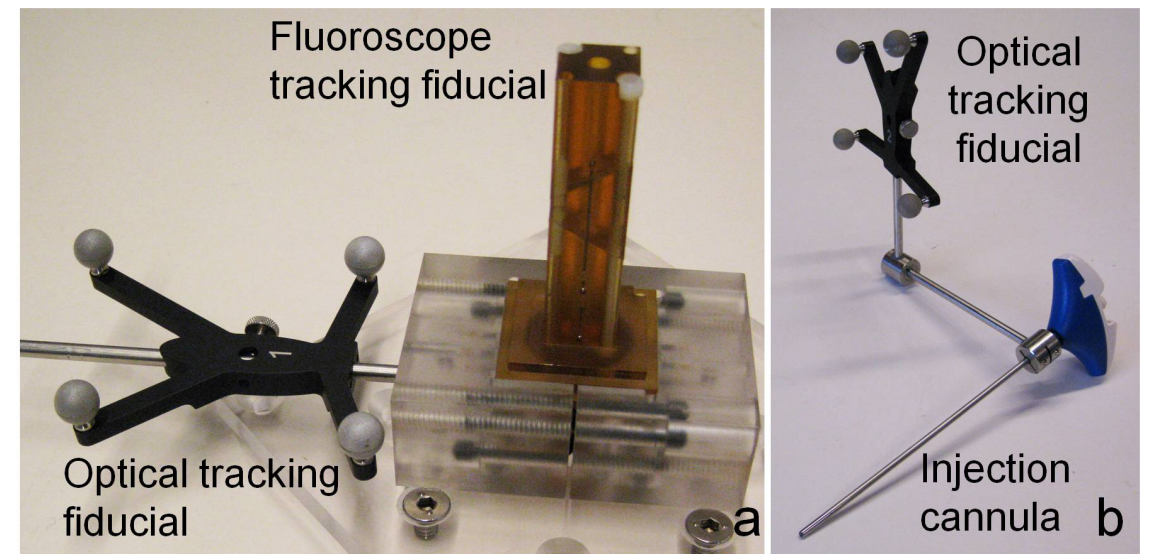

Figure 3. The custom devices used in the proposed system. (a) Optically-tracked fluoroscope fiducial. (b) Optically-tracked injection device. 
computed by least-squared approximation.

\subsection{Intraoperative navigation}

In order to track the patient-mounted hybrid fiducial and the injection device in real-time during the injection, a Polaris (Northern Digital Inc., Waterloo, ON) optical tracker was used. The body of the $18 \times 18 \times 72 \mathrm{~mm}$ FTRAC [6] was made of polycarbonate and contained nine stainless steel beads, and stainless steel wires in four line and two elliptical shapes. It was designed for estimating the 6DOF pose of the fluoroscopy detector from its projection image using the known geometry of each feature. We defined the coordinate system of the optically-tracked rigid body $\left(F_{r b}\right)$ (see Figure 2 ) and the FTRAC $\left(F_{F T R A C}\right)$. The pose of the fluoroscopy detector coordinate with respect to the FTRAC coordinate system $\left(F_{\text {detector,FTRAC }}\right)$ was estimated by maximizing the similarity measure between the virtually projected image
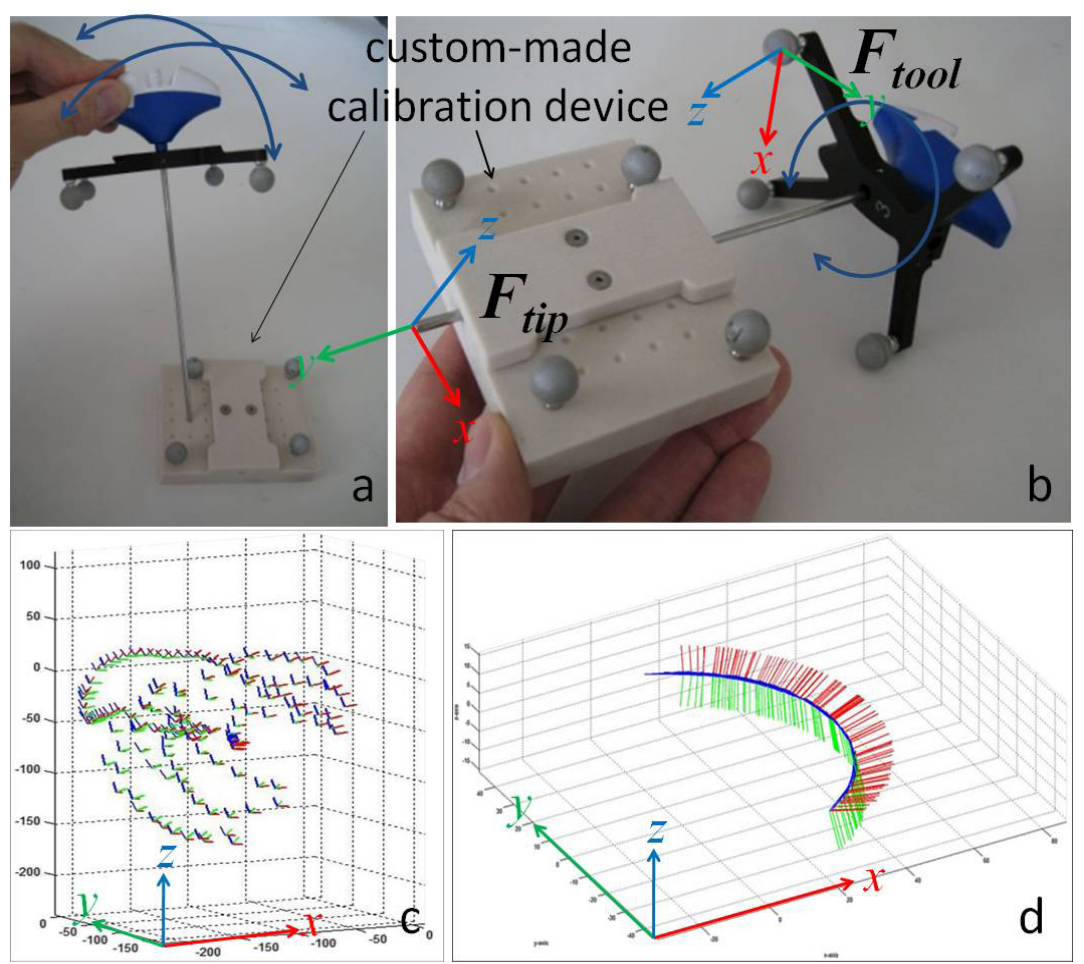

Figure 4. Injection tool calibration. a) pivoting calibration. b) needle axis calibration. c-d) plot of the transformation recorded during the calibration

of the CAD model and the captured C-Arm image. From this transformation and preoperatively calibrated transformation $F_{F T R A C, r b}$, the $F_{\text {detector, } r b}$ was computed for each captured image. By using intensity-based 2D/3D registration of the preoperative $\mathrm{CT}$ and the $\mathrm{C}$-Arm images, the preoperative $\mathrm{CT}$ coordinate with respect to the detector coordinate $\left(F_{\text {detector }, C T}\right)$ was estimated.

The intensity-based 2D/3D registration was conducted by iteratively generating a Digitally Reconstructed Radiograph (DRR) based on CT data and the given transformations between detector and the FTRAC $\left(F_{\text {detector,FTRAC }}\right)$. In order to enhance the speed of generating the DRR, a tetrahedral mesh representation of the target anatomy was used, (described in detail in our prior publication [8-10]). Then, the mutual information between the DRRs and fluoroscope images was optimized to yield the maximum mutual information using a downhill simplex algorithm. Details of this method are described in [7]. An example result of the 2D/3D registration was shown in Figure 5.

Based on the transformation acquired by calibration, intraoperative registration and intraoperative measurement using an
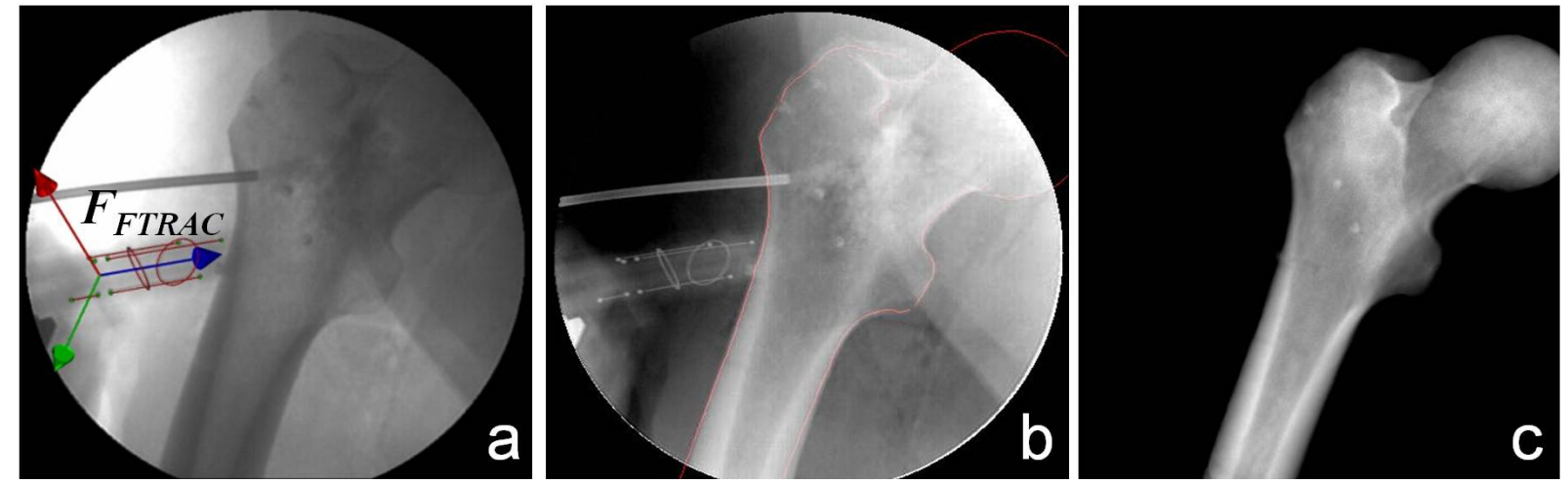

Figure 5. Intensity-based 2D/3D registration. (a) Estimation of the pose of the fluoroscope detector with respect to the FTRAC mounted on the patient. Estimated FTRAC position is overlaid on the C-Arm image (b) Result of the registration. Red edge indicates the position of the registered CT. (c) DRR (Digitally Reconstructed Radiograph) created from preoperative CT. 
optical tracker $\left(F_{n d i, r b}, F_{n d i, t o o l}\right.$ in Figure 2), the tip position and orientation of the injection tool relative to the CT coordinate was computed during the injection as the following.

$$
F_{C T, \text { tip }}=\left(F_{\text {detector }, C T}\right)^{-1} F_{\text {detector }, F T R A C} F_{F T R A C, r b}\left(F_{n d i, r b}\right)^{-1} F_{n d i, t o o l} F_{\text {tool,tip }}
$$

The movement of the injection tool relative to the patient's CT data was displayed graphically for the surgeon (Figure 6). The custom software was implemented with open source tool kits, VTK (The Visualization Toolkit, Kitware, Inc.), Qt (Nokia) and CISST library (the Johns Hopkins University).

Several functions for intraoperative navigation, summarized in Figure 6 (c-e), were implemented. The position of the injection tool was shown in 3D space on the surgical User Interface (UI) as well as 2D MPR (Multi-Planar Reslicing) images. Additionally, the patient position with respect to the fluoroscopy image was displayed using real-time volume rendering of preoperative $\mathrm{CT}$ data. Current tool orientation relative to the planned injection path was displayed using a cone-shaped indicator along the planned path (Figure 6(e)). An image from the viewpoint of the tip of the injection tool was displayed in a sub-window in real-time. Circles on the lateral surface of the cone aligned concentrically when the tool was on planned path.

\subsection{Intraoperative evaluation of the injection}

Injection of the augmentation material was conducted in several batches (typically two to five injections of $5 \mathrm{ml}$ each). In order to achieve an optimized injection, we updated the preoperatively created injection plan according to the surgical progress after each injection. To acquire sufficient information for updating the injection plan, five to six fluoroscopy images were acquired from different directions and the 3D distribution of the injected material was estimated from the images. Subsequent injections were conducted using the updated plan.
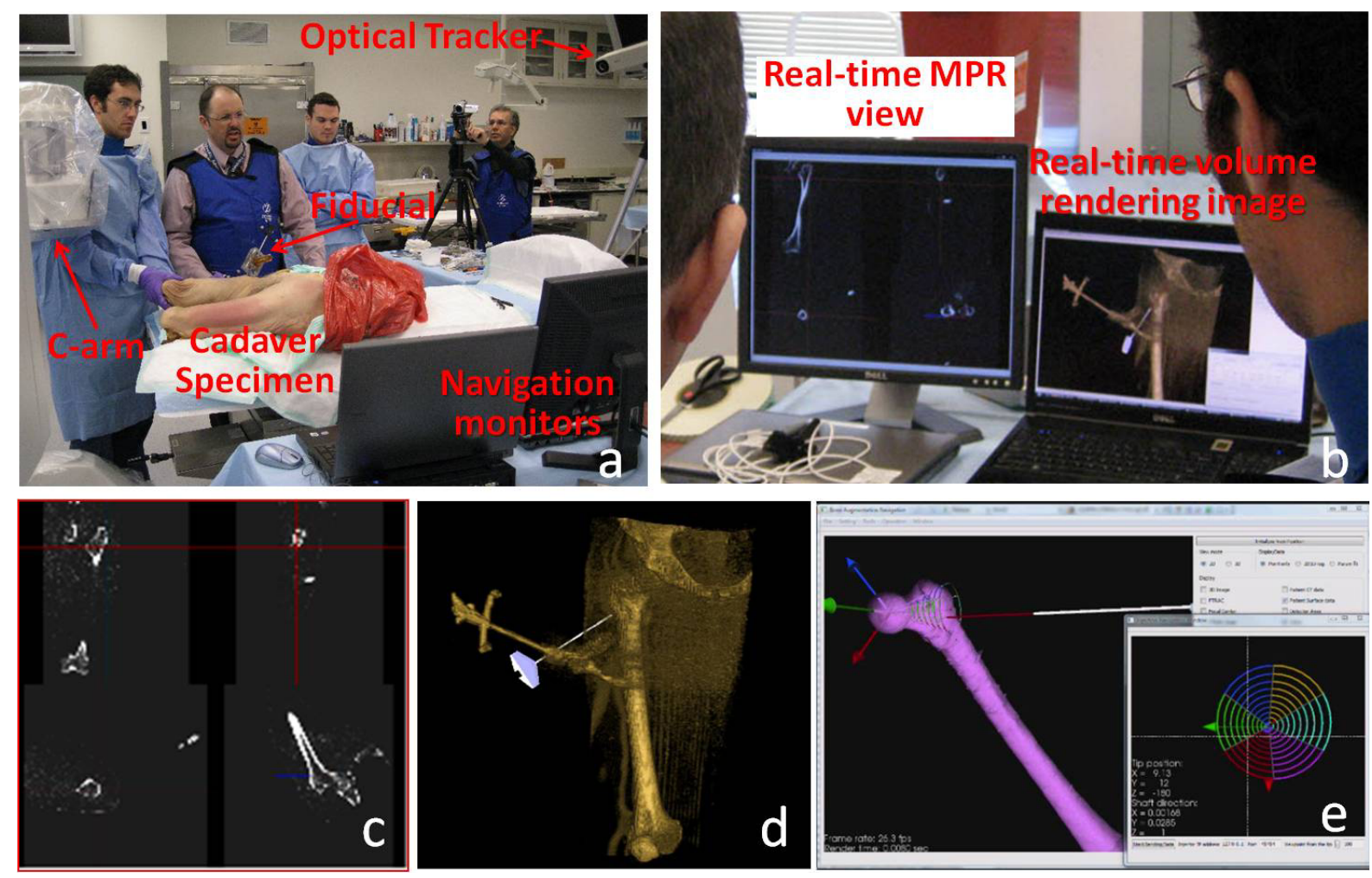

Figure 6. Initial cadaver testing. a) experimental setup, b) view from surgeon's viewpoint, c)-e) navigation functions. c) realtime Multi-Planar Reslicing (MPR), d) visualization of the injection tool with respect to native anatomy, e) needle angle navigation using a cone-shaped guidance 

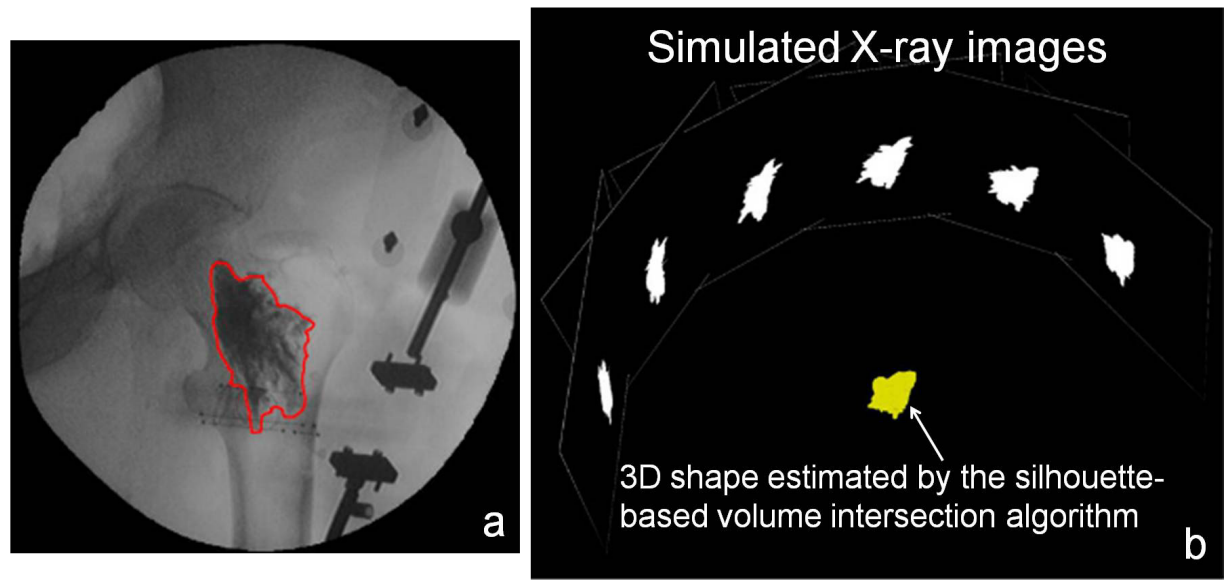

Figure 7. Reconstruction of the injected cement using silhouette-based volume intersection method. (a) X-ray image acquired after injection. Red edge indicates the extracted contour of the cement region. (b) Simulated X-ray image position and estimated distribution of the augmentation material.

1) $3 \mathrm{D}$ shape estimation of the injected material using $2 \mathrm{D}$ projection images

We estimated the 3D injected volume shape using a silhouette-based volume intersection algorithm, which estimates 3D shape from a series of 2D photo images [11-13]. The method was applied to contours of the augmentation material extracted from the C-Arm images, acquired after each injection. First, positions of each image with respect to the world coordinate were estimated using the X-ray fiducial. The contour of the augmentation material was segmented in each image using a semi-automatic region growing algorithm. Based on the position of the series of images and the contours, the 3D shape was reconstructed using a volume intersection algorithm, enhanced for speed by using an octree data structure described previously [14]. Figure 7 demonstrates the method and an example result.

\section{2) Validation of the shape estimation algorithm}

The shape estimation algorithm proposed here was validated using a series of C-Arm images of an augmented femur specimen. Augmentation material was injected into an extracted cadaveric femur bone, then, six C-Arm images from different viewpoints at 30-degree intervals were acquired by a conventional C-Arm with an X-ray image

intensifier. The

specimen was also

scanned by diagnostic

CT to obtain a ground truth shape of the injected material for validation.

Two types of validation studies were conducted: a simulation study using simulated X-ray projection images of the ground truth shape and a study with the real C-Arm images (Figure 8).

The goal of the simulation study was to clarify the
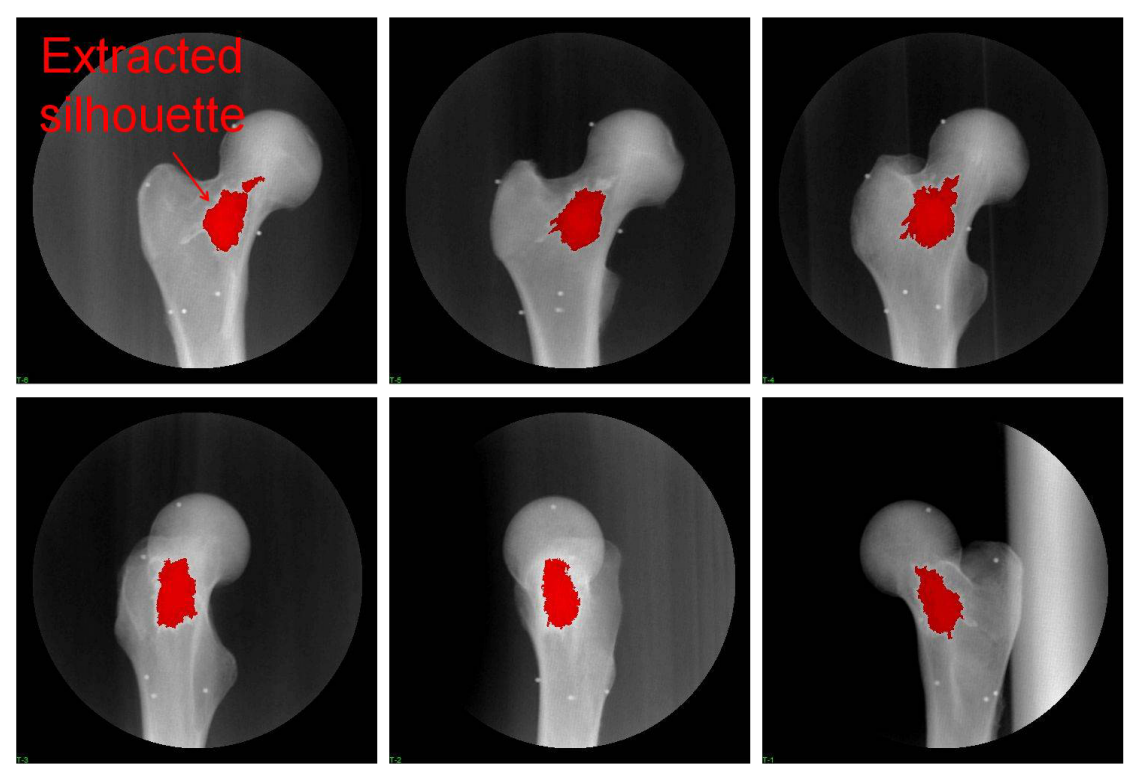

Figure 8. Shape reconstruction from the real C-Arm images. Segmentated regions were shown in red. 
relationship between the number of X-ray images and the accuracy of the estimated shape in respect to the realistic shape of the augmentation material. First, the volumes of the shape reconstructed from CT data $\left(V_{C T}\right)$ and estimated by silhouette-based algorithm assuming a very dense C-Arm scan (360 degrees at 1 degree interval) $\left(V_{\text {best }}\right)$ were computed. One of the well-known limitations of the silhouette-based shape estimation algorithm is inability to reconstruct concave regions. Therefore $V_{\text {best }}$ was considered as the best estimation for the specific shape using our method. Next, by the silhouette-based algorithm, the volume of the augmentation material $(V)$ was estimated under various conditions shown in Figure 10. Then the normalized differences between $V$ and $V_{C T}\left(E_{C T}\right), V_{\text {best }}\left(E_{\text {best }}\right)$ were computed as the following respectively.

$$
E_{C T}=\frac{\left|V-V_{C T}\right|}{V_{C T}}, E_{\text {best }}=\frac{\left|V-V_{\text {best }}\right|}{V_{\text {best }}}
$$

In this simulation study, we assumed that the X-ray source was located at exactly the center at the detector and at the distance of $920 \mathrm{~mm}$ in front of the image plane (Figure 9 left-top). The virtual X-ray projections were collected by rotating C-Arm on the 3 planes as shown in Figure 9 (left-bottom): transverse plane, which was perpendicular to the craniocaudal axis of the patient, a plane angled 30 degrees cranially and 30 degrees caudally through a medio-lateral axis. These planes were chosen with the intention to simulate clinically feasible C-Arm scanning orientations. The detector traveled on a circle of $200 \mathrm{~mm}$ radius centered at the patient's femur as shown in Figure 9 (right).

Real C-Arm images were used to confirm the feasibility of our method in a clinical setting. Six images taken at 30 degrees intervals were first rectified with the method described in 2.1. Then the volume of the augmentation material $(V)$ was computed in the same way as the above-mentioned method and computed $E_{C T}$ as explained in equation (2).

\section{RESULTS}

\subsection{Phantom study for accuracy evaluation}

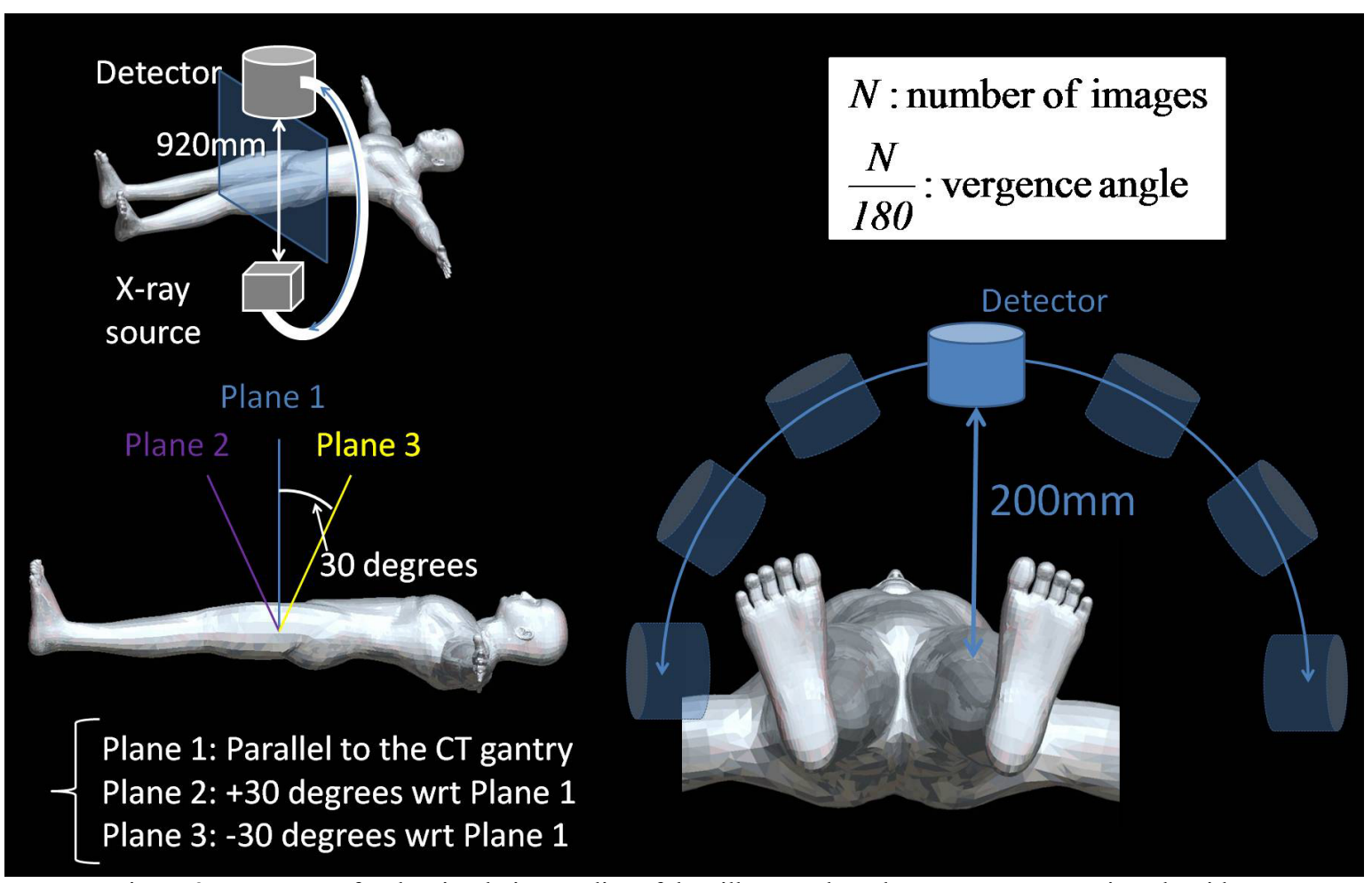

Figure 9. Parameters for the simulation studies of the silhouette-based cement reconstruction algorithm 


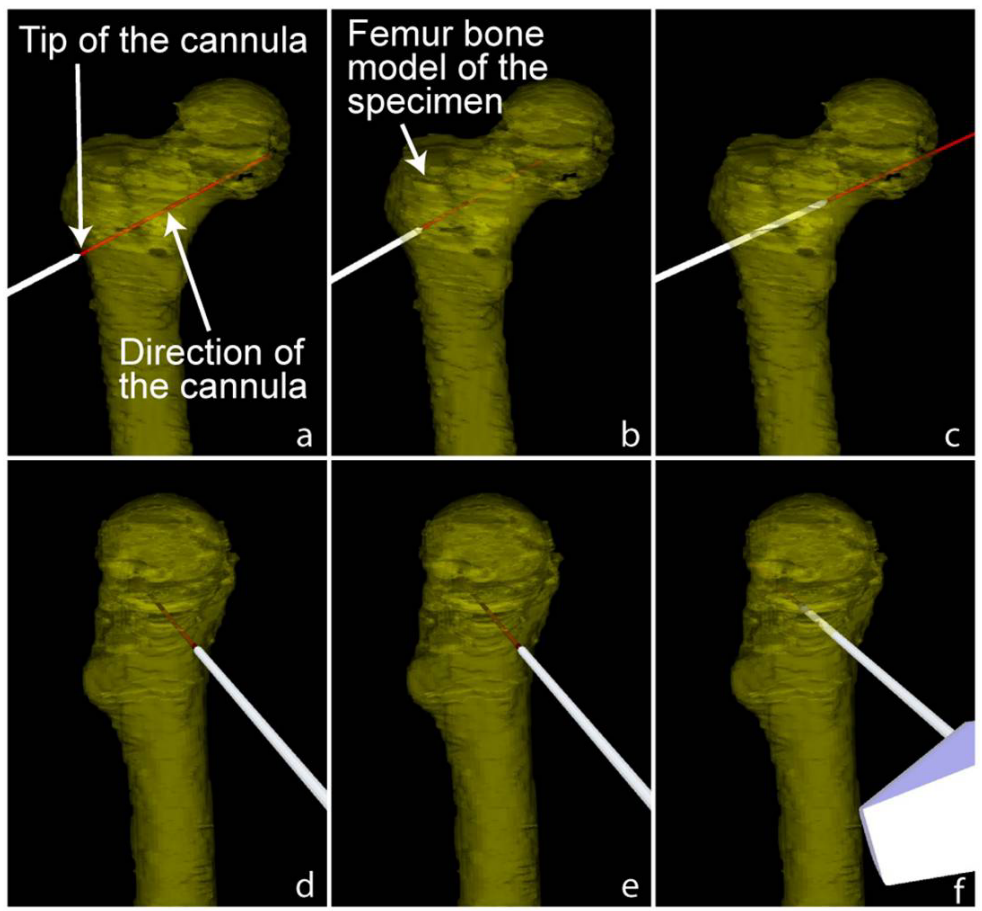

Figure 10. Results of the measurement of the cannula movement during the injection. (a)-(c) Views from frontal side. The tip position and the direction of the shaft were displayed with respect to the femur. Images were shown time sequentially from (a) to (c). (d)-(f) Views from lateral side at the same timeframe as (a)-(c) respectively. (white line: current cannula axis, red line: extrapolated line of the cannula axis.)

In order to evaluate the overall accuracy of the registration between the hybrid-fiducial and the patient coordinate system including tool calibration accuracy and intraoperative registration accuracy, an experiment using a plastic bone phantom
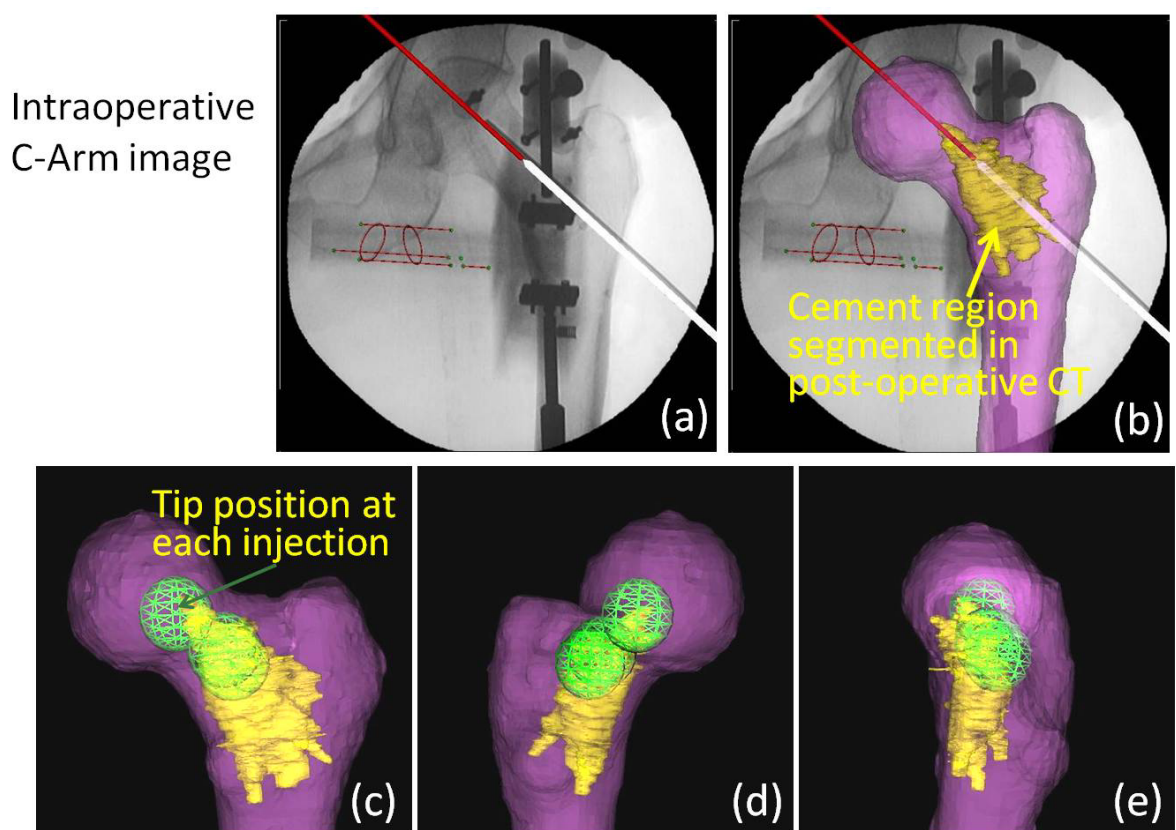

Figure 11. Result of the cadaver study. (a) C-Arm image before the first injection. Tracked cannula model was overlaid onto the C-Arm image, (b) result of 2D/3D registration. Cement region that is segmented in post-operative CT was depicted. (c)(e) relative position between the tip of the injector at each batch and the cement region (see text for more detail) 
was conducted. The experiment showed that the average target registration error at the center of the femoral head was $1.4 \mathrm{~mm}$ and the average rotational error between the coordinate systems was 0.8 degrees when using two images taken from a 35 degree vergence angle. Details of the experiments were reported in our prior paper [5].

\subsection{Cadaver studies}

Three cadaver studies were conducted. Qualitative feedback from the surgeon after the initial cadaver test (Figure 6) suggested that the navigation system successfully located the insertion point and improved performance to precisely control the injection device and follow the planned trajectory. Figure 10 shows the result of the recorded movement of the injection tool during injection of the augmentation material. Figure 11 demonstrates the result of our second cadaver study. The orientation and position of the injection tool was successfully registered to the patient's femur bone (purple in the figure) using intraoperative C-Arm images. The yellow region indicates the distribution of the injected augmentation material which is segmented in the post-operative CT. The three green spheres in the bottom figures indicate the tip position of the injector for each batch (three batches were injected during this surgery), and the volume of the sphere corresponds to the amount of injected material for each batch $(5 \mathrm{ml})$. The relationship between the tip position at the injection and the resulting shape of the injected material can be clearly seen in the image. This result implies that the injected material flowed in a caudal direction in the patient's femur bone from the injection point.

Figure 12 compared the $\mathrm{CT}$ images obtained prior and after injection in the third cadaver study. Since the augmentation material contained contrast agent, the injected material could be clearly identified in high contrast in the slice images.

The 3D distributions of augmentation material acquired from postoperative CT data in our 3 cadaver studies were shown in Figure 13. Major differences in characteristics of the shape of distribution for each specimen were observed.

\subsection{Evaluation of shape estimation of the augmentation material}

Figure 14 shows an example of the shape reconstruction from 6 simulated X-ray images at the configuration shown in Figure 7. The shapes estimated from only 6 images show a comparable result with the reconstruction from the CT image. Results of our simulation studies are shown in Figure 15 and 16. The specimens (a)-(c) in the figure correspond to the shapes in Figure 14. Figure 16 shows the computed $E_{\text {best }}$ and $E_{C T}$ (equation 2) with respect to the number of the

\section{Before injection}

After
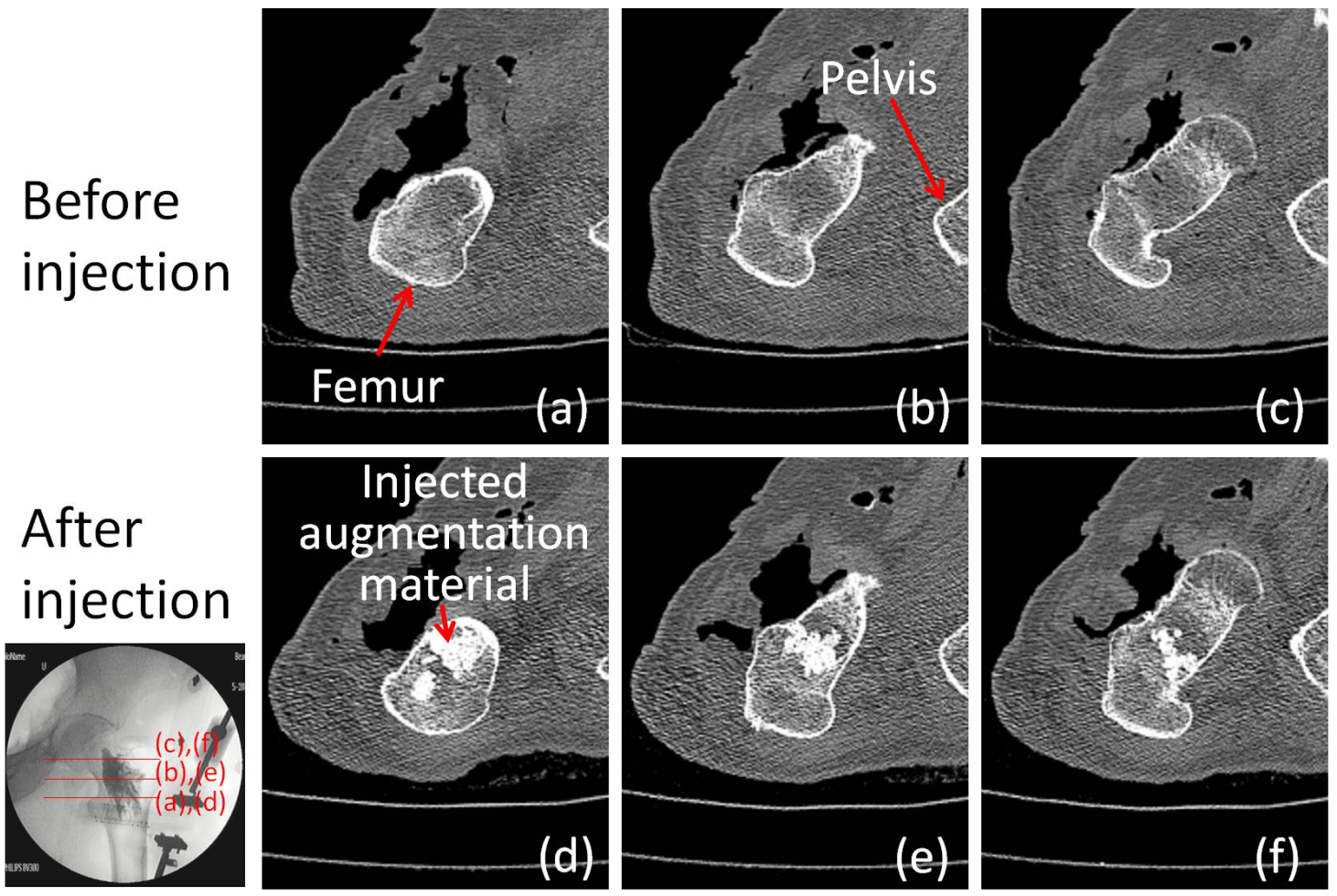

Figure 12. CT data acquired at before and after injection. Injected augmentation material can be clearly identified in high contrast. (left bottom) position of each slice. 

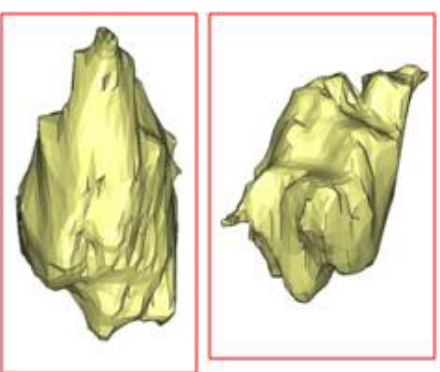

Specimen (a)
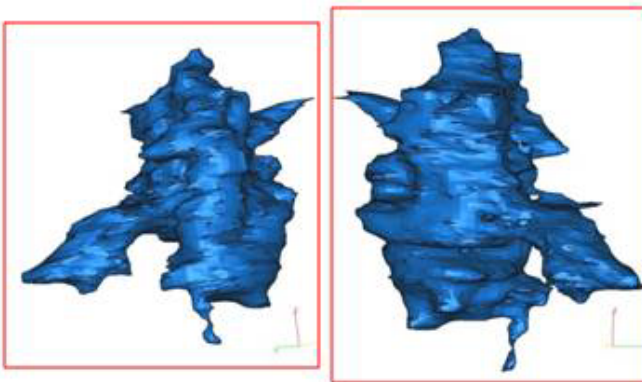

Specimen (b)
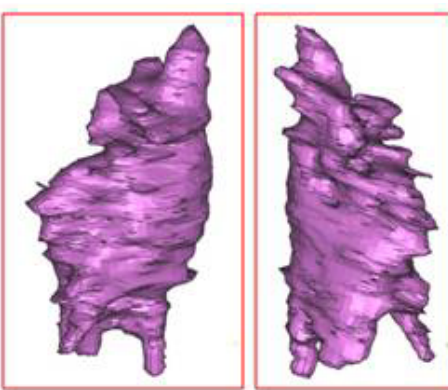

Specimen (c)

Figure 13. 3D shapes of the augmented material in the three cadaver studies. Views from two different viewpoints for each specimen were shown.

simulated X-ray images. In all specimens the estimated volume approached the best case estimation $\left(V_{\text {best }}\right)$ as the number of images increased in all specimens. Figure 16 shows that the estimated volume approached the best case estimation regardless of scan plane. Specimen (c) was used in this simulation.

In the shape estimation study using real C-Arm images (Figure 8), the estimated volume of the augmentation material was $3,511 \mathrm{~mm}^{3}$, whereas the volume measured in CT data (ground truth) was $6,159 \mathrm{~mm}^{3}$; thereby the normalized ratio $E_{C T}$ was $43 \%$.

\section{DISCUSSIONS AND CONCLUSIONS}

This paper has reported the design and initial cadaveric evaluations of our image-guided femoroplasty system. The intraoperative registration using intensity-based 2D/3D registration was achieved using a custom optically-tracked fluoroscopic fiducial. A silhouette-based volume intersection algorithm was used to estimate the 3D shape of the injected augmentation material.
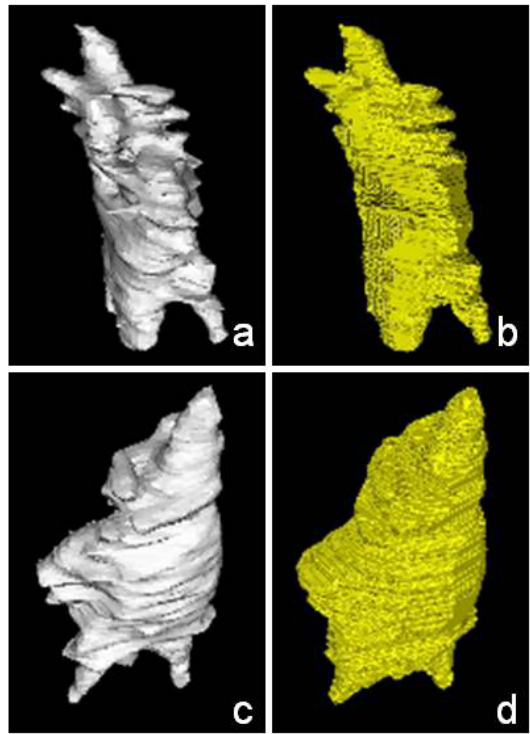

Figure 14. An example of the shape reconstruction from 6 simulated X-ray images (configuration was shown in figure 7). (a)(c) reconstruction from CT (ground truth), (b)(d) reconstruction from 6 silhouettes. (a)

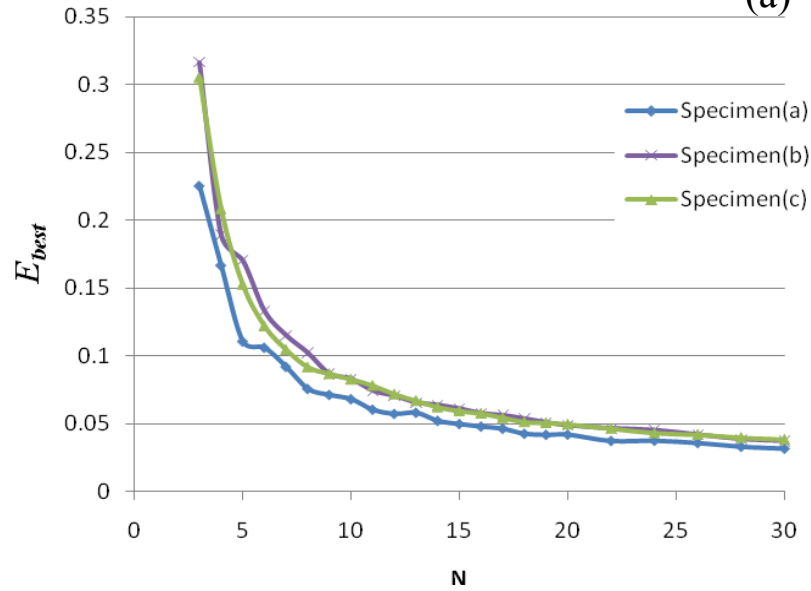

(b)

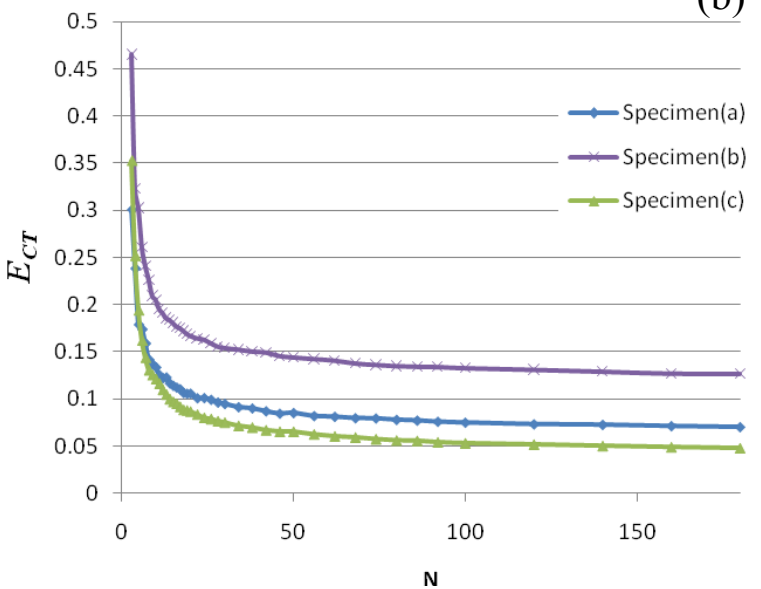

Figure 15. Comparison of the silhouette-based shape estimation between specimens. Assumed that detector was moved on the plane 1 (see Figure 10) (a) number of images vs. $E_{\text {best }}(\mathrm{b})$ number of images vs. $E_{C T}$ 
(a)
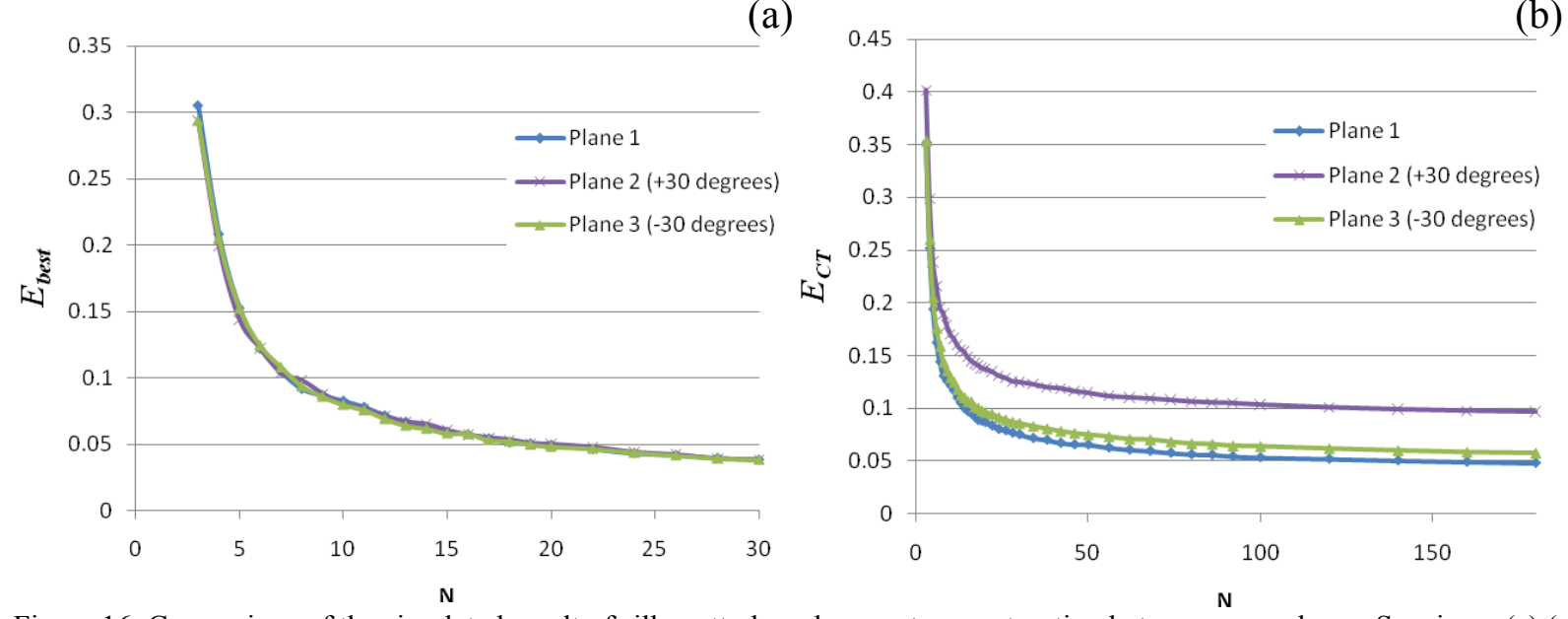

Figure 16. Comparison of the simulated result of silhouette-based cement reconstruction between scan planes. Specimen (c) (see Figure 14) was used for the simulation. (a) number of images vs. $E_{\text {best }}$ (b) number of images vs. $E_{C T}$

The cadaver studies demonstrated efficacy and accuracy of the navigation system. Qualitative feedback from the surgeon suggested that the intuitive navigation display provided the ability to resolve geometric uncertainties associated with the preoperative injection plan and the patient during injection. The simulation studies showed that the distribution of the injected augmentation material could be reasonably reconstructed using six X-ray projection images.

One alternative approach to estimate the 3D distribution of the injected material during surgery is to acquire intraoperative CT data using a state-of-the-art C-Arm system in which a motion encoder, a flat-panel detector and dedicated software are installed to obtain 3D reconstruction from a number of 2D cone-beam projection images. While the intraoperative CT would have potential to provide an accurate measurement of the injected cement, the amount of ionizing radiation dose is higher than the conventional approach and the acquisition time required for the scan and 3D reconstruction could induce adverse effects. Therefore, in this research we have pursued a radiologically less invasive and less time-consuming way which provides a reasonable measurement result. A modified approach using intraoperative $\mathrm{CT}$, while maintaining the lower radiation dose, is planned as future work.

Results of the simulation study (Figure 15) suggest that the volume of the injected augmentation material was estimated within about $12 \%$ error when 6 images were used (graph a). In specimen (b), $12 \%$ of the volume was overestimated even if the scan was very dense (graph b). This result indicates that the shape of the injected material of the specimen (b) contains large concavities, which intrinsically precludes accurate reconstruction using the silhouette-based method, compared to the other two specimens. Further analysis on the relationship between the shape characteristic and the minimum theoretical error would improve the algorithm. Figure 16 (b) indicates that errors in the very dense scan (180 images) differs only $5 \%$ between the scan planes, which implies that the choice of the scan plane does not largely affect the result.

The result of the experiment using real C-Arm images showed significant underestimation by the silhouette-based method. Our current implementation uses simple binary contour information extracted from the X-ray projection image and computes the intersection volume over all the images; therefore the estimated volume becomes highly sensitive to the subtle error in the pose estimation of each image, segmentation and imperfect camera parameter calibration. The pose estimation error causes error of the direction of the viewing volumes, which ends up decreasing the intersecting volume. The segmentation and the camera calibration error cause the same effect in the result. In order to minimize the effect of these instabilities during the data acquisition process, we are planning to modify our method using a level-set representation $[15,16]$, which is a probabilistic approach to compensate for the ambiguity induced by the data acquisition error.

Although the current approach will not reconstruct an exact shape, it has the potential to accurately estimate the location of the injected material with respect to the femur, and approximate the boundary of the augmentation material. We believe that this information may be beneficial by providing a targeted region of interest for more precise low-dose tomographic reconstruction [17]. 
This paper describes the development and experimental analysis of a prototype image-guided surgery system for femoroplasty. Our future plans also include making the devices compact and integrating the robotic hand-held injection device so that the injection itself can be robotically controlled according to the current position of the injection device.

\section{ACKNOWLEDGEMENT}

This research has been financially supported by NIH5R21EB007747-02 and JSPS Postdoctoral Fellowships for Research Abroad.

\section{REFERENCES}

[1] Beckmann J, Ferguson SJ, Gebauer M, Luering C, Gasser B, Heini P. , "Femoroplasty--augmentation of the proximal femur with a composite bone cement--feasibility, biomechanical properties and osteosynthesis potential," Med.Eng.Phys. Sep 29(7), 755-764 (2007).

[2] Heini PF, Franz T, Fankhauser C, Gasser B, Ganz R. , "Femoroplasty-augmentation of mechanical properties in the osteoporotic proximal femur: a biomechanical investigation of PMMA reinforcement in cadaver bones,"

Clin.Biomech.(Bristol, Avon) Jun 19(5), 506-512 (2004).

[3] Sutter EG, Mears SC, Belkoff SM. , "A biomechanical evaluation of femoroplasty under simulated fall conditions," J. Orthop. Trauma. (in Press).

[4] Basafa E, Armiger RS, Kutzer DM, Sutter E, Mears S, Armand M. , "Optimizing cement injection in osteoporotic femur augmentation," Proceedings of 9th Annual Meeting of CAOS-International, Boston, June, 319-322 (2009).

[5] Otake Y, Armand M, Sadowsky O, Kutzer DM, Armiger RS, Basafa E, et al. , "Development of a navigation system for femoral augmentation using an intraoperatie C-arm reconstruction," Proceedings of 9th Annual Meeting of CAOSInternational, Boston, June, 177-180 (2009).

[6] Jain AK, Mustafa T, Zhou Y, Burdette C, Chirikjian GS, Fichtinger G. , "FTRAC--a robust fluoroscope tracking fiducial," Med.Phys. Oct 32(10), 3185-3198 (2005).

[7] Sadowsky O, Lee J, Sutter EG, Wall SJ, Prince JL, Taylor RH. , "Enhancement of mobile c-arm cone-beam reconstruction using prior anatomical models," Proceedings of SPIE Conference on Medical Imaging, Orlando, March 7258, 5B (2009).

[8] Sadowsky O, Cohen JD, Taylor RH. "Rendering tetrahedral meshes with higher-order attenuation functions for digital radiograph reconstruction," Visualization, 2005. VIS 05. IEEE , 303-310 (2005).

[9] Sadowsky O, Cohen JD, Taylor RH. , "Projected tetrahedra revisited: a barycentric formulation applied to digital radiograph reconstruction using higher-order attenuation functions," IEEE Trans.Vis.Comput.Graph. Jul-Aug 12(4), 461-473 (2006).

[10] Sadowsky O, Chintalapani G, Taylor RH. , "Deformable 2D-3D registration of the pelvis with a limited field of view, using shape statistics," Med.Image Comput.Comput.Assist.Interv.Int.Conf.Med.Image

Comput.Comput.Assist.Interv. 10(Pt 2), 519-526 (2007).

[11] Kutulakos KN, Seitz SM. , "A Theory of Shape by Space Carving," International Journal of Computer Vision 07/01 38(3), 199-218 (2000).

[12] Prakash S, Robles-Kelly A. , "A semi-supervised approach to space carving," Pattern Recognit 2 43(2), 506-518 (2010).

[13] Starck J, Hilton A. , "Virtual view synthesis of people from multiple view video sequences," Graphical Models 11 67(6), 600-620 (2005).

[14] Szeliski R. , "Rapid Octree Construction from Image Sequences," CVGIP: Image Understanding 7 58(1), 23-32 (1993).

[15] Osher S, Sethian JA. , "Fronts propagating with curvature-dependent speed: Algorithms based on Hamilton-Jacobi formulations," Journal of Computational Physics 11 79(1), 12-49 (1988).

[16] Varshney KR, Paragios N, Deux JF, Kulski A, Raymond R, Hernigou P, et al. , "Postarthroplasty examination using X-ray images," IEEE Trans.Med.Imaging Mar 28(3), 469-474 (2009).

[17] Zou Y, Pan X, Sidky EY., "Image reconstruction in regions-of-interest from truncated projections in a reduced fanbeam scan," Phys.Med.Biol. 50(1), 13-27 (2005). 